\title{
Cytomorphologic Features of Gastric-Type Endocervical Adenocarcinoma in Liquid-Based Preparations
}

\author{
Joerg Schwock ${ }^{a, b} \quad$ Blerta Starovac Zanobia F. Khand Jelena Mirkovic ${ }^{\text {b, e }}$ \\ Carlos Parra-Herran ${ }^{f}$ Hyang Mi Ko ${ }^{a, b}$ Marjan Rouzbahman ${ }^{a, b}$ Zeina Ghorabb, e \\ aDivision of Pathology, University Health Network, Toronto, ON, Canada; ${ }^{b}$ Department of Laboratory Medicine \\ and Pathobiology, University of Toronto, Toronto, ON, Canada; 'Department of Laboratory Medicine, William \\ Osler Health System, Brampton, ON, Canada; ${ }^{d}$ Department of Pathology, Lakeridge Health, Oshawa, ON, Canada; \\ eDepartment of Pathology, Sunnybrook Health Sciences Centre, Toronto, ON, Canada; 'Department of Pathology, \\ Brigham and Women's Hospital, Boston, MA, USA
}

\section{Keywords}

Cervix - Gynecologic cytology · Gastric type · Papanicolaou test - Endocervical adenocarcinoma - Liquid-based cytology $\cdot$ Morphometry

\section{Abstract \\ Objective: Gastric-type endocervical adenocarcinoma (GAS) is a recently described, uncommon, and aggressive tumor with distinct morphologic features and HPV-independent etiology. Data on GAS in liquid-based cytology (LBC) Papani- colaou (Pap) test preparations from a North American pa- tient population are scant. We systematically assessed the cytomorphologic characteristics of GAS in LBC from patients in Ontario and examined if glandular cell nuclear area could represent a readily assessable feature which may aid in GAS detection. Study Design: Pap test slides preceding the diag- nosis of GAS were retrieved locally or requested from outside laboratories. A structured review of 15 cytomorphologic fea- tures was performed using the available LBC Pap test slides of GAS and a set of usual-type endocervical adenocarcino- mas (UEA). Morphometry of the glandular cell nuclear area was performed, and normalized values were compared to UEA and benign endocervical cells. Results: At least 1 Pap}

test (5 ThinPrep ${ }^{\circledR}, 11$ SurePath $^{\oplus}$, and 1 direct smear) was available for 14 patients. Original LBC Pap test diagnoses were negative for intraepithelial lesion or malignancy (NILM) (7), adenocarcinoma/carcinoma (6), atypical glandular cells (2), and adenocarcinoma in situ (1). Review detected abnormal glandular cells in 6/7 NILM cases. Honeycomb-like sheets, nuclear enlargement, and microvesicular cytoplasm were the single most common architectural, nuclear, and cytoplasmic features, respectively. Microvesicular cytoplasm (100 vs. $17 \%)$, honeycomb-like sheets ( 87 vs. $8 \%$ ), prominent nucleoli ( 93 vs. $25 \%$ ), and anisonucleosis ( 93 vs. $50 \%$ ) were most discriminatory for GAS versus UEA, respectively. Yellow mucin, intranuclear cytoplasmic pseudoinclusions, and goblet/Paneth-like cells were uncommon, but unique for GAS. Glandular cell nuclear area normalized to neutrophils was found to be significantly increased in GAS compared to benign endocervical cells. Conclusions: GAS is under-recognized and may mimic reactive endocervical cells. Awareness

Marjan Rouzbahman and Zeina Ghorab contributed equally as cosenior authors. Findings of this study were presented in part at the 2019 Annual Meeting of the United States and Canadian Academy of Pathology at National Harbor, Maryland (Abstract\# 1855, platform presentation).
C) 2020 The Author(s)

Published by S. Karger AG, Basel

This article is licensed under the Creative Commons AttributionNonCommercial-NoDerivatives 4.0 International License (CC BYNC-ND) (http://www.karger.com/Services/OpenAccessLicense) Usage and distribution for commercial purposes as well as any distribution of modified material requires written permission.
Joerg Schwock

Department of Laboratory Medicine and Pathobiology, Laboratory Medicine Program University Health Network Toronto General Hospital, University of Toronto 200 Elizabeth Street, Room 11E-215B, Toronto ON M5G 2C4 (Canada) joerg.schwock@uhn.ca 
of the tumor type and its cytomorphology is critical for early detection. Identification of glandular cells with uniform nuclear enlargement in conjunction with any of the other cytologic features may help avoid false-negative Pap results. Neutrophils may serve as convenient size reference and visual aid.

(c) 2020 The Author(s)

Published by S. Karger AG, Basel

\section{Introduction}

Mucinous carcinoma of gastric-type (also termed gastric-type adenocarcinoma [GAS]) is a variant of endocervical adenocarcinoma which is recognized in the latest WHO classification [1]. In contrast to endocervical adenocarcinoma of usual-type (UEA), GAS is primarily characterized by a human papilloma virus (HPV)-independent etiology, pyloric gland-type mucin production (detectable by immunohistochemistry with HIK1083 and MUC6 antibodies), and unfavorable clinical behavior [1]. GAS manifests as part of a spectrum of endocervical lesions which comprise benign, premalignant, and malignant entities and, in case of the latter, encompass a well-differentiated form historically termed "minimal deviation adenocarcinoma" (MDA, also adenoma malignum) $[1,2]$. GAS has recently been confirmed as the major subtype in the HPV-independent category of endocervical adenocarcinomas and requires separation from HPV-related mucinous tumors which encompass signetring cell type, intestinal type, invasive stratified mucinproducing, and mucinous carcinoma, not otherwise specified [3]. Extant knowledge about GAS is limited. This is partly due to the fact that the diagnostic category "endocervical mucinous adenocarcinoma" was used in the past for a heterogeneous group of neoplasms. MDA represents a morphologically challenging diagnosis whose bland features may have been overemphasized in relation to its benign and precursor mimics $[2,4,5]$. On the other hand, the proportion of "overt" adenocarcinoma permissible for MDA subcategorization is not well defined. GAS is associated with Peutz-Jeghers syndrome, an autosomal dominant disorder characterized by STK11 tumor suppressor gene germline mutations [6]. STK11 variants are also detectable in a subset of sporadic tumors $[7,8]$. Additional alterations in TP53, POLE, CDKN2A/B, mismatch repair genes, Fanconi anemia genes, KRAS, $B R C A 2$, and ARID1A were recently identified [8].

The histopathologic diagnosis of GAS rests primarily on the morphologic identification of (a) voluminous clear and/or pale eosinophilic cytoplasm and (b) distinct

GAS in Liquid-Based Cytology cytoplasmic borders of the neoplastic cells [9]. Ancillary studies, including immunohistochemistry and molecular methods, to support lack of HPV infection are used for confirmation. Specific features associated with GAS and its precursor lesions in Papanicolaou (Pap) tests, in particular for liquid-based cytology (LBC), have not been well established. Consequently, clinically significant abnormalities may be overlooked during the screening process or misinterpreted as reactive due to lack of familiarity with the cytologic manifestation of the disease resulting in delayed clinical management.

Importantly, with introduction of HPV testing as a primary means of screening, GAS developing sporadically in a woman not otherwise clinically suspected to be at risk (as opposed to an individual suffering from known or suspected Peutz-Jeghers syndrome which predisposes to GAS spectrum abnormalities) will even less likely be detected before becoming clinically symptomatic. Although early diagnosis and treatment are key to improving the prognosis of GAS, a meta-analysis focused on MDA found low detection rates both by cytology and biopsy, thus often requiring multiple successive sampling procedures [10]. Notably, clinical observation of cervical thickening and radiologic detection of multicystic cervical lesions and/or hypertrophy by ultrasound, computed tomography, or magnetic resonance imaging may aid in case identification but cannot reliably distinguish between benign and early malignant changes $[2,10,11]$. MRI features described as "cosmos" sign (small cysts or solid parts surrounded by larger cysts) may represent lobular endocervical glandular hyperplasia (LEGH), a benign morphologic mimic of MDA and potential precursor lesion (identifiable as "atypical" LEGH) of gastric-type mucinous carcinomas $[2,11,12]$.

The cytologic features of GAS were recently examined in a systematic comparison with UEA based on 14 cases represented in conventional Pap smear preparations [9]. Descriptions of GAS in LBC preparations are few [13-16]. Thus, our study was aimed to examine the features of GAS in Pap tests from a North American patient cohort with focus on LBC preparations which represent the most common preparation type in our practice. In addition, we hypothesized that uniform nuclear enlargement may be a reproducible characteristic which, in conjunction with other cytomorphologic features, may aid in the assignment of an appropriate reporting category (i.e., atypical glandular cells (AGC) or adenocarcinoma, as indicated) to initiate further clinical management. 


\section{Materials and Methods}

Case Selection and Retrieval

Research ethics board approval was obtained. Pap test slides of 16 patients identified with GAS diagnosis rendered on surgical pathology specimens between January 2000 and July 2018 were retrieved from the authors' institutions or requested from outside laboratories. Twelve patients were part of a prior study [8]. Slides for 2 patients were not provided (excluded). HPV testing was performed for 12 of the remaining 14 patients by Linear Array ${ }^{\circledR} \mathrm{HPV}$ Genotyping (Roche Diagnostics, Laval, QC) $(n=11)$ or Hybrid Capture HC ${ }^{\circledR} 2$ (Digene, Gaithersburg, MD, USA) $(n=1)$. P16 immunohistochemistry was performed as previously described [8]. Twelve (6 ThinPrep ${ }^{\circledR}[\mathrm{TP}]$ and 6 SurePath $\left.^{\circledR}[\mathrm{SP}]\right)$ UEA and $12(6$ TP and 6 SP) negative for intraepithelial lesion or malignancy (NILM) cases were used as control materials. UEA diagnoses were confirmed by histology (biopsy or resection). All cases were reviewed by pathologists with expertise in gynecologic pathology.

\section{Cytomorphologic Evaluation}

Two staff cytopathologists and a fellow (J.S., B.S., and Z.G.) independently reviewed the Pap tests. Slides with identifiable abnormalities were examined for 15 predetermined cytomorphologic variables divided into 3 categories: (1) architectural (flat, honeycomb-like sheets, and peripheral feathering), (2) nuclear (abrupt anisonucleosis [ $\geq 1: 3$ nuclear size variation of neighboring cells], nuclear enlargement [ $>$ the size of a neutrophil], round-ovoid nuclear shape [as opposed to elongated], prominent nuclear membrane irregularities ["raisinoid"], prominent nucleoli [ $\geq$ the size of a neutrophil nuclear lobe], vesicular [iso-/hypochromatic] chromatin, and intranuclear pseudoinclusions and mitotic figures), and (3) cytoplasmic ("foamy," microvesicular cytoplasm, neutrophil phagocytosis, goblet/Paneth cell features, yellow cytoplasmic mucin, and cytoplasmic globules). Disagreements were resolved by consensus.

Cytomorphometric Analysis

Microscopic images (tagged image file format) were taken using a DFC450 camera mounted on a DMR microscope and Leica Application Suite Version 4.12.0 (Heerbrugg, Switzerland). Depending on thickness, 2-16 separate $\mathrm{z}$-stack images were captured of at least 3 representative cell groups per slide and processed using Helicon Focus Version 7.0.2 Software (Kharkiv, Ukraine). Morphometry of the glandular cell nuclear surface area (GAS, UEA, and NILM) was performed using Aperio ImageScope (Sausalito, CA, USA). Z-stacks were inspected to ensure the largest nuclear surface area was measured. Measurement of 15 glandular cell nuclei and 3-5 neutrophils per slide was used to generate a normalized glandular cell nuclear/neutrophil area ratio. The KruskalWallis test was used for statistical comparison (GraphPad Prism, La Jolla, CA, USA).

\section{Results}

\section{Study Population and Cytologic Test Results}

Seventeen Pap test slides from 14 patients were available for review either from the authors' institutions (6/17; $35 \%$ ) or from external laboratories within the province of
Table 1. Case summary

\begin{tabular}{|c|c|c|c|}
\hline Patients & Preparation & Original diagnosis & $\begin{array}{l}\text { Review } \\
\text { result }\end{array}$ \\
\hline 1 & $\mathrm{TP}$ & AdenoCa, NOS & AdenoCa \\
\hline 2 & $\mathrm{TP}$ & AdenoCa, endocervical & AdenoCa \\
\hline 3 & $\mathrm{TP}$ & AdenoCa, endocervical & AdenoCa \\
\hline 4 & $\mathrm{TP}$ & AdenoCa, endocervical & AdenoCa \\
\hline 5 & $\mathrm{TP}$ & Carcinoma & AdenoCa \\
\hline 6 & SP & AGC, favor neoplasia & AdenoCa \\
\hline 7 & $\mathrm{SP}$ & AdenoCa & AdenoCa \\
\hline 8 & $\begin{array}{l}S P-1 \\
S P-2\end{array}$ & $\begin{array}{l}\text { ASCUS, AGC } \\
\text { AIS }\end{array}$ & $\begin{array}{l}\text { AGC } \\
\text { AdenoCa }\end{array}$ \\
\hline 9 & SP & NILM & AGC \\
\hline 10 & $\mathrm{SP}$ & NILM & AGC \\
\hline 11 & $\mathrm{SP}$ & NILM & AGC \\
\hline 12 & $\begin{array}{l}S P-1 \\
S P-2 \\
S P-3\end{array}$ & $\begin{array}{l}\text { NILM } \\
\text { NILM } \\
\text { NILM }\end{array}$ & $\begin{array}{l}\text { AGC } \\
\text { AGC } \\
\text { AGC }\end{array}$ \\
\hline 13 & SP & NILM & NILM \\
\hline 14 & Direct smear & AdenoCa, endocervical & AdenoCa \\
\hline
\end{tabular}

TP, ThinPrep ${ }^{\circledR}$; SP, SurePath ${ }^{\circledR}$; AdenoCa, adenocarcinoma; AGC, atypical glandular cells; AIS, adenocarcinoma in situ; ASCUS, atypical squamous cells of undetermined significance; NILM, negative for intraepithelial lesion or malignancy; NOS, not otherwise specified.

Ontario (Table 1). Multiple Pap tests were available for review from 2 patients (case \#8: $n=2$; case \#12: $n=3$ ). The interval between these Pap tests ranged between 4 and 26 months. A single Pap test was reported concurrent with the surgical pathology specimen. For the remaining cases, the median interval between sign out of the closest Pap test result and the surgical pathology specimen was 75 days (range: $4-480$ days). The median patient age was 59.5 years at the time of histopathologic diagnosis (range: 35-79 years). In 4/14 patients, the histopathologic diagnosis was rendered on small specimens (i.e., biopsy and curetting) while the majority had large resection specimens (radical or simple hysterectomies). In 11/14 patients, the subtype diagnosis of GAS was either rendered or suggested in the original diagnostic histopathology report. In 3 cases (all dating before 2010), GAS was diagnosed upon review. None of the cases was classified as 
Table 2. Cytomorphologic features of GAS and UEA identified in LBC preparations

\begin{tabular}{|c|c|c|c|}
\hline & Features & $\begin{array}{l}\text { GAS } \\
N(\%)\end{array}$ & $\begin{array}{l}\text { UEA } \\
N(\%)\end{array}$ \\
\hline \multirow[t]{2}{*}{ Architecture } & Flat, honeycomb-like sheets* & $13(87)$ & $1(8.3)$ \\
\hline & Peripheral feathering* & $4(27)$ & $7(58)$ \\
\hline \multirow[t]{8}{*}{ Nuclear } & Prominent nucleoli ( $\geq$ neutrophil nuclear lobe)* & $14(93)$ & $3(25)$ \\
\hline & Vesicular, iso- to hypochromatic chromatin* & $14(93)$ & $8(58)$ \\
\hline & Nuclear enlargement (>neutrophil) & $15(100)$ & $12(100)$ \\
\hline & Round-ovoid nuclear shape & $14(93)$ & $11(92)$ \\
\hline & Abrup $t \geq 1: 3$ anisonucleosis & $14(93)$ & $6(50)$ \\
\hline & Irregular nuclear membranes & $8(53)$ & $8(58)$ \\
\hline & Mitotic figures & $4(27)$ & $6(50)$ \\
\hline & Intranuclear pseudoinclusions & $3(20)$ & $0(0)$ \\
\hline \multirow[t]{5}{*}{ Cytoplasm } & Microvesicular, foamy cytoplasm* & $15(100)$ & $2(17)$ \\
\hline & Neutrophil entrapment/phagocytosis* & $5(33)$ & $4(33)$ \\
\hline & Yellow mucin* & $3(20)$ & $0(0)$ \\
\hline & Goblet/Paneth-like cells & $3(20)$ & $0(0)$ \\
\hline & Mucin globules & $1(6.7)$ & $5(42)$ \\
\hline
\end{tabular}

GAS, gastric-type endocervical adenocarcinoma; UEA, usual-type endocervical adenocarcinoma; LBC, liquid-based cytology. ${ }^{*}$ Cytomorphologic features previously examined by Kawakami et al. [9].

MDA. P16 immunohistochemistry was performed for all tumors and showed focal/patchy reactivity in 3 (21\%). A negative p16 result was obtained in the remaining cases. HPV was undetectable in all cases tested $(n=14)$ which included those with p16 reactivity.

Sixteen Pap test slides were LBC preparations (5 TP and $11 \mathrm{SP}$ ), and one was a conventional direct smear which reflects the predominant use of LBC for Pap testing in our geographic area. The direct smear Pap test, which was positive for adenocarcinoma at the time of original reporting and upon review, was excluded from further analysis in order to focus on the cytomorphologic findings associated with GAS in LBC. All TP preparations originated from a single academic center. The remaining slides originated from both academic- and communitybased laboratories. The LBC tests were originally reported with the following interpretations: "adenocarcinoma/ carcinoma": $n=6$, "AGC/AGC favor neoplasia": $n=2$, "adenocarcinoma in situ" (AIS): $n=1$, and "NILM": $n=$ 7. Retrospective review of these slides (although not blinded with respect to the final histopathologic diagnosis) confirmed the features of adenocarcinoma in $8 / 16$ tests (including 1 slide originally reported as AGC and 1 slide originally reported as AGC favor neoplasia). In 6/7 tests (corresponding to 5 patients) with an original NILM interpretation, review revealed abnormal glandular cells potentially reportable as AGC or higher. In 1 Pap slide, no evidence of abnormality was identifiable (NILM confirmed). If preparation types were taken into account, an unequivocal interpretation of malignancy (adenocarcinoma, carcinoma, or AIS) was rendered on $5 / 5 \mathrm{TP}$ versus 2/11 SP slides.

\section{Cytomorphologic Analysis of GAS Features}

Systematic analysis of the architectural, nuclear, and cytoplasmic features in 15 LBC Pap tests with identifiable glandular abnormalities revealed honeycomb-like sheets $(13 / 15,87 \%)$, nuclear enlargement to a size $>$ a neutrophil $(15 / 15,100 \%)$, and "foamy," microvesicular cytoplasm $(15 / 15,100 \%)$ as the most frequently observed single characteristic in each category, respectively (Table 2). Additional features identified in $>50 \%$ of the cases were prominent nucleoli, iso- to hypochromatic chromatin (compared to background cells), round to ovoid nuclear shape, abrupt anisonucleosis, and prominently irregular nuclear membranes. Neutrophil phagocytosis, peripheral feathering, mitotic figures, intranuclear cytoplasmic pseudoinclusions, goblet and/or Paneth-like cells, yellow mucin, and cytoplasmic globules were identified in a minority. Similar architectural, cytoplasmic, and nuclear findings were identifiable in TP and SP preparations (Fig. 1). 

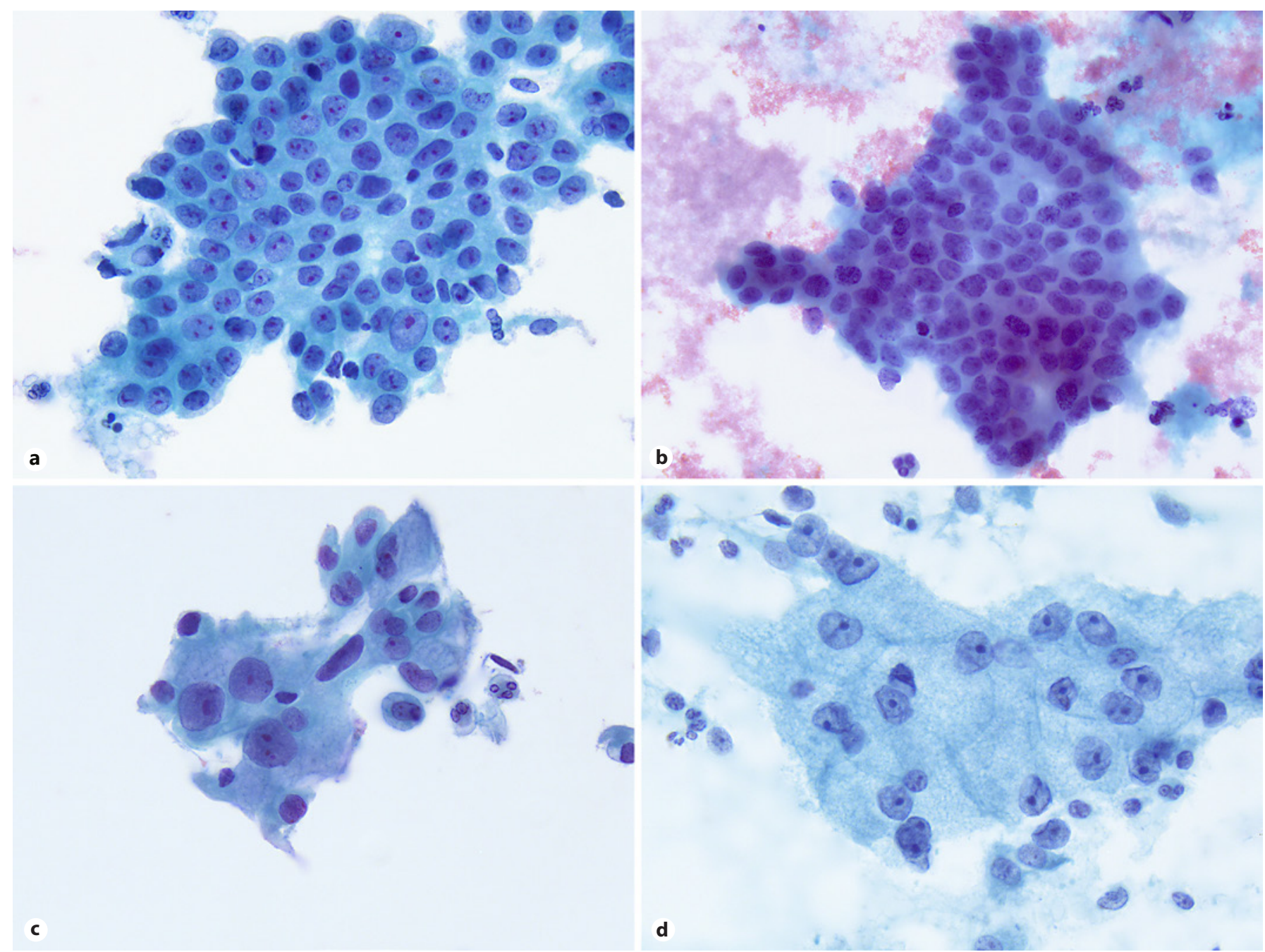

Fig. 1. Common cytomorphologic characteristics of GAS in TP and SP cytology preparations. a, b Honeycomblike sheets of glandular cells with increased nuclear size. Note the irregular spacing of the nuclei in gastric-type adenocarcinoma which is similar to the "drunken" arrangement known for pancreatobiliary carcinoma. c, d Microvesicular, foamy cytoplasm of the abnormal glandular cells (TP (a, c); SP (b, d); ×63, oil immersion). GAS, gastric-type endocervical adenocarcinoma; TP, ThinPrep ${ }^{\circledR}$; SP, SurePath ${ }^{\circledR}$.

Figure 2 illustrates the features of GAS in comparison with UEA and benign endocervical cells in SP. Using the same set of features, comparison with UEA revealed microvesicular cytoplasm, honeycomb-like architecture, prominent nucleoli, and abrupt anisonucleosis (100 vs. $17 \%, 87$ vs. $8 \%, 93$ vs. $25 \%$, and 93 vs. $50 \%$ ) as most discriminatory for GAS versus UEA, respectively. Yellow mucin (20\%), intranuclear cytoplasmic pseudoinclusions (20\%), and goblet/Paneth-like cells (20\%), although uncommon, represented unique features identified in GAS only (Fig. 3). Intracytoplasmic neutrophil entrapment/ phagocytosis was also observed in a minor portion of the UEA cases.

\section{Cytomorphometric Analysis of GAS Nuclear Area}

GAS can mimic benign and/or reactive endocervical epithelium through honeycomb-like architecture and cytoplasmic mucin content (as opposed to UEA which is most frequently mucin depleted). We hypothesized that nuclear enlargement, as one of the most prevalent features, could aid in the cytologic identification of GAS. Thus, we sought to obtain more observer-independent, objective data using morphometric measurements of the nuclear area in comparison with cases reported as NILM and UEA. To circumvent the variability inherent to cytologic preparations, glandular cell nuclear/neutrophil area ratios were generated. Endocervical cells of NILM cases
60

Acta Cytologica 2021;65:56-66 DOI: $10.1159 / 000511003$
Schwock/Starova/Khan/Mirkovic/ Parra-Herran/Ko/Rouzbahman/Ghorab 


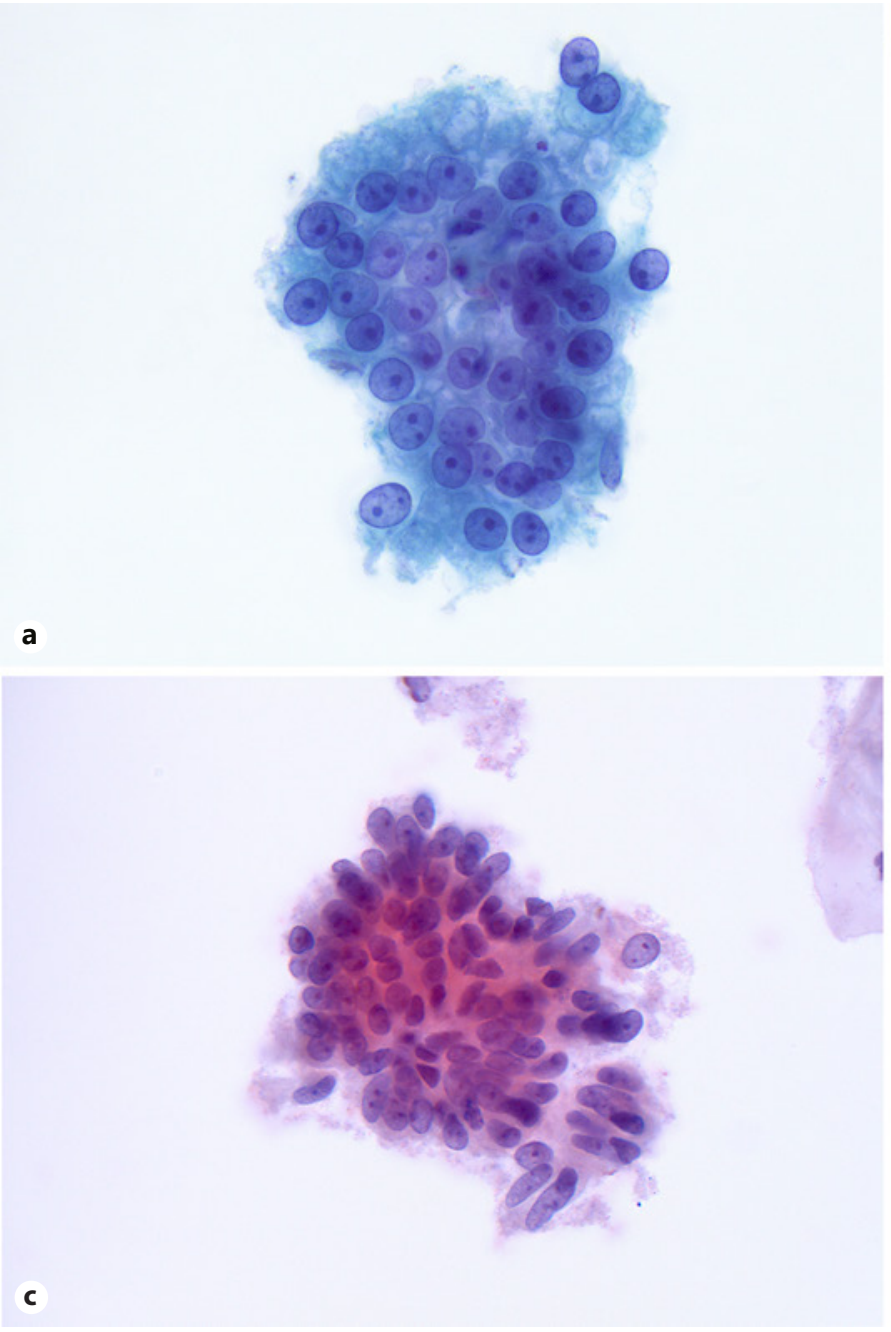

had a nuclear area similar to the neutrophils (mean ratio: 0.99 , median ratio: 0.98 , and standard deviation [SD]: $0.19)$. In contrast, UEA and GAS showed significantly larger nuclei with a mean ratio, median ratio, and SD of $1.56 / 2.12,1.57 / 2.00$, and $0.33 / 0.52$, respectively (Fig. 4 ).

\section{Discussion}

A meta-analysis of MDA (a tumor now considered part of the GAS spectrum) found vaginal discharge followed by irregular or contact bleeding as the most frequent presenting complaints [10]. Both clinical complaints commonly serve as clinical triggers for a diagnostic Pap test. Currently, most cytologic descriptions of GAS rely on case reports or small series with few of these publications emanating from North America [17]. Thus, the cytologic findings of GAS (and its precursors), in par-

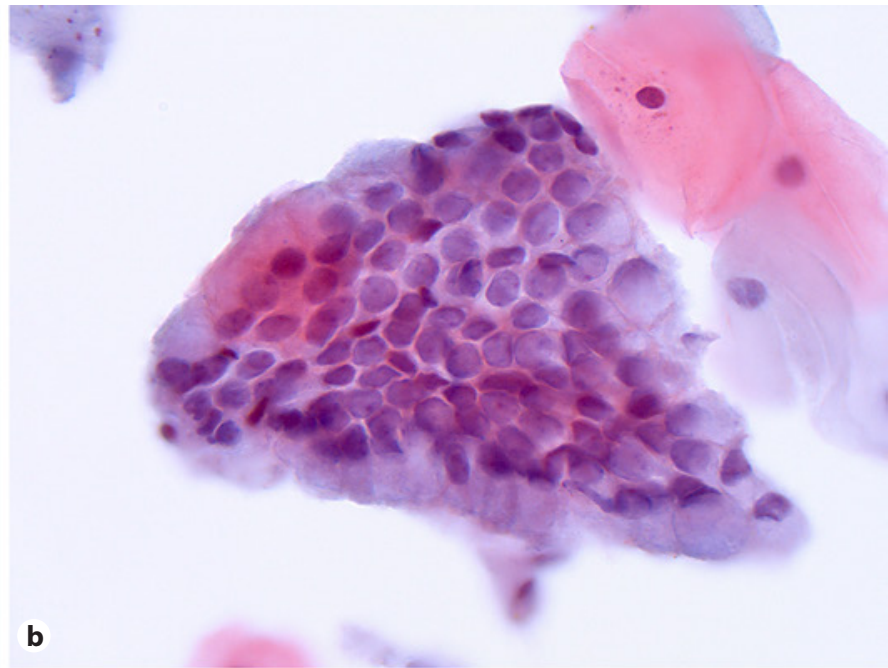

Fig. 2. Comparison between benign endocervical cells, GAS, and UEA. GAS (a) displays a cell arrangement somewhat similar to benign endocervical cells (b) but distinct from UEA (c) which may result in misinterpretation. Nuclear features including uniform nuclear enlargement firmly place both GAS and UEA into the neoplastic category (TP; $\times 63$, oil immersion). GAS, gastric-type endocervical adenocarcinoma; UEA, usual-type endocervical adenocarcinoma; TP, ThinPrep ${ }^{\circledR}$.

ticular in LBC preparations, are insufficiently defined. However, early detection is critical in this poor prognosis tumor type $[18,19]$. Atypical LEGH and gastric-type AIS may represent precursors to GAS [20-23]. Recent reports based on a small number of cases indicate that early detection of these precursor lesions in cytology may be possible $[24,25]$. Low (cyto-)pathologist awareness of the subtype and a perception that the cytologic detection may be difficult or even impossible may both contribute to low rates of timely detection and erroneous classification as endometrial carcinoma [16]. A recent publication by Kawakami et al. [9] who systematically studied the cytologic features of GAS in comparison with UEA in direct smear preparations provided a framework for further exploration. We examined if cytomorphologic features that might indicate the presence of a neoplastic process (including features previously described for GAS in direct smear preparations) are identifiable in LBC preparations 


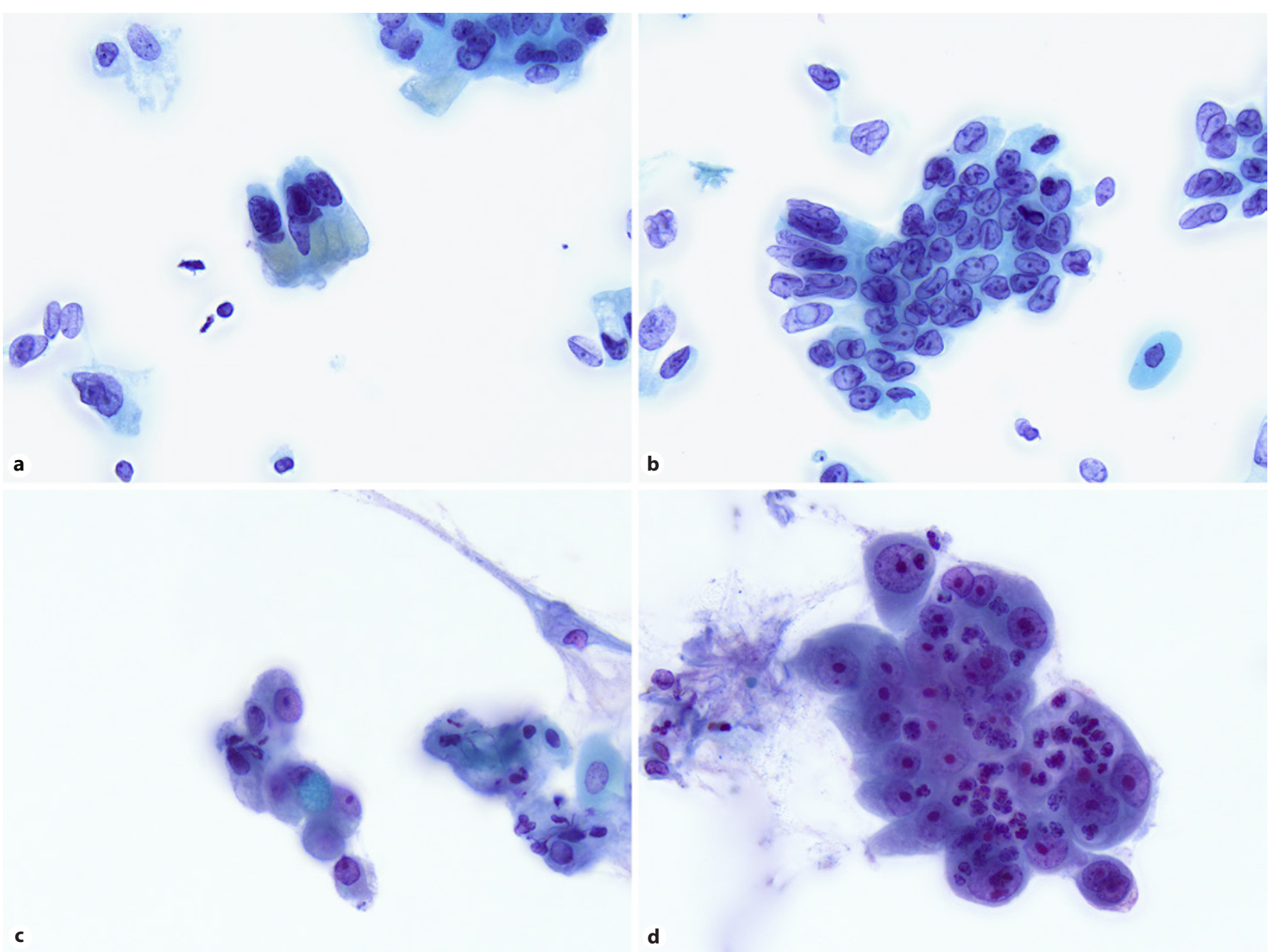

Fig. 3. Cytomorphologic characteristics of GAS seen in a minority of cases. a Yellow cytoplasmic mucin (SP). b Intranuclear cytoplasmic inclusion (SP). c Paneth-like neuroendocrine cell (TP). d Intracytoplasmic neutrophil entrapment/phagocytosis (TP) (×63, oil immersion). GAS, gastric-type endocervical adenocarcinoma; TP, ThinPrep $^{\circledR} ;$ SP, SurePath ${ }^{\circledR}$.

(TP and SP) from a North American patient cohort diagnosed with GAS.

The golden-yellow color of gastric/pyloric gland-type mucin in Pap-stained cytology preparations has been reported as one of the main features associated with gastrictype glandular lesions [26, 27]. Golden-yellow mucin was seen by Kawakami et al. [9] in 6/14 cases of their series (ranging from mild to prominent). However, it is important to note that golden-yellow mucin is also present in the context of benign gastric-type glandular proliferations [27]. In addition, Omori et al. [28] state that "it is difficult to recognize yellow mucin on LBC because the mucin color becomes paler." In their report, Omori et al. [28] nicely illustrate the difference in mucin color (yellow vs. pink in normal endocervix) in an image which also demonstrates the relatively uniform enlargement of the nuclei compared to the normal endocervical cells. Lu et al. [15] report that golden-yellow intracytoplasmic mucin was not noticeable in their series of 11 patients with pretreatment Pap tests (both TP and SP) out of a cohort of 23 GAS cases indicating that, indeed, golden-yellow mucin may not be a particularly sensitive feature for GAS detection, at least in LBC. Of note, only 4/11 Pap tests in their study were originally reported as AGC (favor neoplasia or NOS) while the remaining results were NILM, HSIL, and unsatisfactory, thus underlining the difficulty of GAS detection in cytology [15]. Yuan et al. [24] note that although golden-yellow mucin was seen, it was rela- 


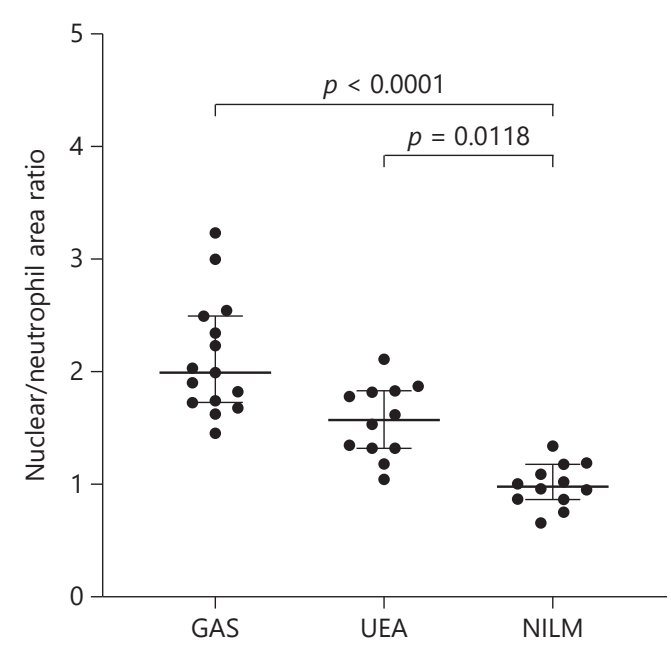

Fig. 4. Normalized nuclear area of GAS, UEA, and benign endocervical cells. GAS cells contain nuclei of approximately twice the area of a neutrophil or a benign endocervical cell. The normalized nuclear areas of both GAS and UEA are similar and significantly different from benign endocervical cells. GAS, gastric-type endocervical adenocarcinoma; UEA, usual-type endocervical adenocarcinoma; NILM, negative for intraepithelial lesion and malignancy (benign endocervical cells).

tively scanty in the atypical cells thought to represent gastric-type AIS in their study. In contrast, Okuyama et al. [25] report golden-yellow mucin predominately on the surface of the glandular cell clusters presumed to represent atypical LEGH. A possible explanation for these seemingly contradictory findings is provided by Yamanoi et al. [29] who found a progressive decrease of MUC6 and, more significantly, of the terminal a1,4-linked $N$ acetylglucosamine residues attached to the MUC6 scaffold across the spectrum of LEGH, atypical LEGH, MDA, and GAS. Their findings suggest the possibility that the detection of this particular tinctorial quality of intracellular mucin in gastric-type lesions may depend on the degree of lesional differentiation rather than the preparation type (i.e., direct smear vs. LBC) used for staining with the Pap method. Indeed, yellow mucin was identified only in 3/15 Pap tests examined in our study while it was not seen in the single direct smear Pap test. In addition, we recently encountered bright golden-yellow cytoplasmic mucin in an LBC preparation of a Brunner gland hamartoma, an intestinal lesion with both HIK1083 and MUC6 reactivity, sampled by endoscopic ultrasoundguided fine-needle aspiration (Fig. 5). Thus, yellow mu-

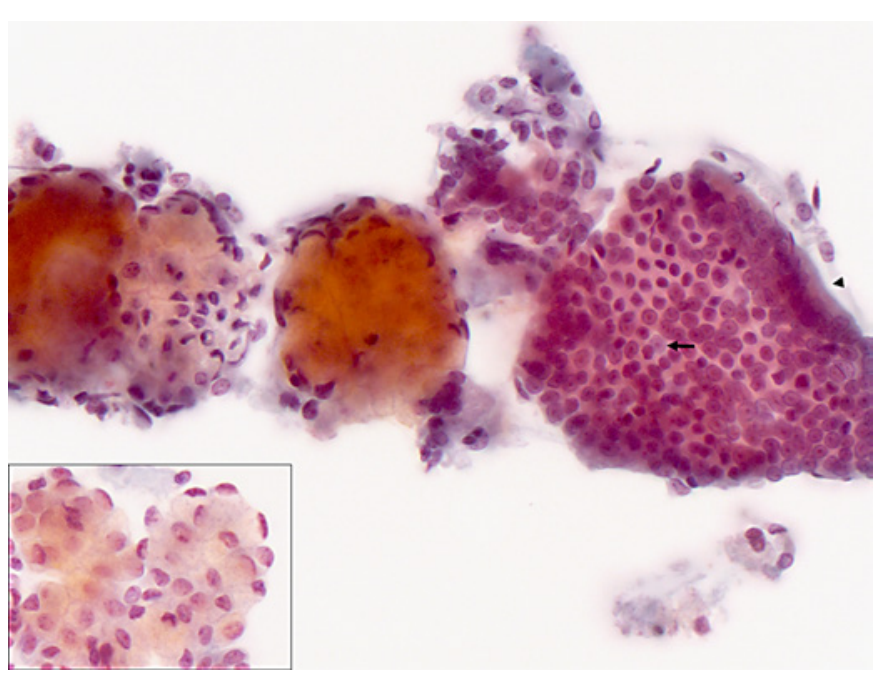

Fig. 5. Yellow cytoplasmic mucin in a TP preparation of a Brunner gland hamartoma. Brunner gland hamartoma is a benign proliferation of duodenal submucosal glands. The mucin-secreting gland epithelium is distinguishable from the intestinal surface epithelium by the abundant yellow cytoplasmic mucin content and lobular architecture which stand in contrast to the goblet cells (arrow) and brush border (arrowhead) in the latter $(\times 40 ; \times 63$ (inset), oil immersion). TP, ThinPrep ${ }^{\circledR}$.

cin most likely represents a feature more prevalent in well-differentiated or benign/premalignant lesions of gastric type which should not be relied upon exclusively for cytologic GAS detection. In contrast, the so-called "background," extracellular mucin may indeed not be as easily detectable in LBC versus direct smears due to preparation-related, technical causes (similar to the diminished presence of necrotic debris). In support, Hissong et al. [14] state that background mucin may clump and cling to tumor cells in TP instead of the diffuse distribution seen in direct smears. Kawakami et al. [9] report moderate to prominent background mucin in all of their GAS cases but also in $55 \%$ of the UEA. As such, in general, the diagnostic usefulness of extracellular mucin is doubtful.

Our findings are novel, in part, due to the focus on nuclear enlargement in GAS which is a feature not previously examined systematically. Historically, Granter and Lee reported 7 MDA cases which had cytologic findings other than normal endocervical cells identified in 5/7 cases after review [30]. Among those findings were "enlarged bland glandular cells" in all 5 cases with honeycomb-like sheets, vacuolated or lacy cytoplasm without increased nucleocytoplasmic ratio, and, importantly, "nuclei [...] enlarged to approximately twice the size of normal endocervical cell nuclei with fine chromatin and small nucle- 
oli or chromocenters" [30]. The authors propose that "if such cells are seen in abundance, especially in large branching sheets, [...] a recommendation for biopsy follow-up might lead to the detection of MDA" [30]. Since normal endocervical cells may not always be present or confidently identifiable, such as in a case with circumferential LEGH or extensive GAS, neutrophils which are typically quite prevalent may represent a more useful internal comparator for size reference. Our finding that, upon review, 6/7 LBC Pap test slides with an original NILM interpretation contained glandular cells which were distinct from benign endocervical epithelium suggests the possibility that, indeed, the abnormality was either overlooked or misinterpreted as reactive. Similar to Kawakami et al. [9], we found honeycomb-like architecture and abundant "foamy" cytoplasm as frequently observed characteristics which, in retrospect, are potential sources of diagnostic confusion with benign endocervical epithelium. While uniform nuclear enlargement of superficially reactive appearing glandular cells (to an extent $>$ the average area of the neutrophils or endocervical cell nuclei in a given Pap test) should give at least cause for close inspection, the identification of additional cytologic features can clinch the diagnosis of adenocarcinoma and, indeed, may provide clues to the presence of a gastrictype lesion.

Yellow mucin, intranuclear cytoplasmic pseudoinclusions, and presence of goblet or Paneth-like cells are uncommon Pap test findings which, in the appropriate clinicopathologic context, may permit a presumptive diagnosis of GAS. Lack of "feathering" arrangement, prominent nucleoli, and vesicular chromatin can serve as additional clues. For the cytopathologist, it may be helpful to be reminded of the cytomorphologic resemblance of GAS with the "foamy gland pattern" seen in adenocarcinomas of the gastrointestinal tract and other anatomical sites [31, 32]. Stewart et al. [32] reported 4 GAS cases and a single "mixed-type," HPV18-positive endocervical adenocarcinoma with "foamy gland" morphology in $30-80 \%$ of the tumor in histology. Pap tests performed for the 3 GAS cases were interpreted as AIS, AGC, and atypical endocervical cells. However, the cytologic correlate for the "foamy gland" morphology was not further examined or illustrated [32]. Precisely, this resemblance with pancreatobiliary carcinoma was emphasized in a recent review [33]

Aside from the already mentioned yellow mucin and the presence of goblet or Paneth-like cells, an infrequent but striking feature in our study was intranuclear cytoplasmic pseudoinclusions. Pseudoinclusions were re- ported in the cytology preparations of 19/24 (79\%) LEGH cases but none of the 4 cases of MDA by Hashi et al. [34]. In our study, this feature was seen in 3 cases only of which one was reported with atypical LEGH as a component of the lesion. Importantly, no intranuclear inclusions were identified in UEA cases suggesting that, similar to yellow mucin and goblet/Paneth-like cells, this feature may be unique to GAS and/or GAS precursor lesions.

Finally, in contrast to Kawakami et al. [9], intracytoplasmic neutrophil entrapment/phagocytosis was identified in only $33 \%$ of our Pap tests while $93 \%$ of the cases in their study displayed this feature. The reason for the difference is currently not known. A potential explanation may be their inclusion of endometrial cytology (11/14 case) samples [9]. Of note, intracytoplasmic neutrophils were also seen in $70 \%$ of the UEAs (which included endometrial cytology in 12/20 cases) which suggests, in agreement with our findings, that this feature is unlikely of much utility in the distinction between GAS and UEA. Intracytoplasmic neutrophils, however, may have some value in establishing the diagnosis of adenocarcinoma or AGC in general terms. Of note, the "bag of polys" appearance known to occur with endometrial adenocarcinomas in Pap test cytology has to be considered as an additional differential diagnosis. As such, intracytoplasmic neutrophils may also represent an additional source of diagnostic confusion between GAS and endometrial carcinoma [16].

Identification of GAS in cytology specimens depends without doubt on the cytopathologist's awareness of the entity and familiarity with the cytomorphologic presentation. The earliest descriptions of the cytologic features (mainly MDA) are available from both Japanese and North American authors [35-37]. DNA content analysis was used by the Japanese groups in an effort to objectively support cytomorphologic findings associated with MDA $[35,36]$. Some features mentioned in these descriptions such as cytoplasmic "tails" reported by Vogelsang et al. [38] and Szyfelbein et al. [37] are likely preparationrelated rather than tumor-type specific. While our report was in preparation, a North American study with $22 \mathrm{cy}-$ tology specimens including 14 cervical and vaginal samples was published [17]. The cytology preparation type(s) used were not described. Indeterminate results (AGC and suspicious) were reported for 7/22 (32\%) cytology specimens in this study from an academic center even though many cases had a prior diagnosis and/or concurrent surgical specimen. Of 5 cytology specimens seen in consultation, only 1 case was reported with a definite diagnosis of adenocarcinoma by the outside pathologist although en-
Schwock/Starova/Khan/Mirkovic/ Parra-Herran/Ko/Rouzbahman/Ghorab 
dometrial rather than endocervical origin was suspected. No difference in tinctorial quality of the mucin was seen in Pap stains, again supporting the findings of our study [17].

In summary, in our series of 16 LBC Pap tests (both TP and SP) of GAS patients from Ontario, glandular cell abnormalities were identifiable in retrospect in 15/16 tests. Honeycomb-like sheets, nuclear enlargement, and microvesicular cytoplasm were the single most commonly observed architectural, nuclear, and cytoplasmic features, respectively. Uniform glandular cell nuclear enlargement approximately twice that of a neutrophil or (if detectable) benign endocervical cell nucleus might represent a useful criterion to trigger further diligent examination of a Pap test for additional features of neoplasia. Cytopathologists may find it helpful to keep in mind that at least the welldifferentiated part of the gastric-type spectrum of endocervical lesions displays "test-tube"-like microarchitectural features similar to benign and reactive endocervical cells and that diligent examination may hold the clue to the nature of the underlying abnormality. Further studies to substantiate our findings are required. In practice, cell block preparations of the residual sample from the LBC Pap test including ancillary studies (although not examined in our study) may support the presumptive cytopathologic diagnosis. A combined Alcian blue/periodic acid-Schiff stain is expected to yield red staining. MUC6 and HIK1083 are expected to show immunohistochemical reactivity, whereas p16 and hormone receptors (estrogen and progesterone) are mostly negative [33]. None of these tests, however, are entirely specific or sensitive, and, as such, integration of cyto-/histomorphologic, radiologic, and clinical data is paramount. Rarely, ancillary studies assume greater importance in cases with equivocal morphology $[39,40]$. A comment may be included in the report, as appropriate, to inform clinicians regarding the aggressive nature of the gastric subtype and to consider genetic counseling for Peutz-Jeghers syndrome if the tumor subtype is confirmed [6]. Finally, a recent study on the transcriptome profile of endocervical adenocarcinomas supports the distinction of usual, intestinal, and gastric subtypes and points towards tight junction disruption in intestinal and gastric adenocarcinomas as a potential molecular mechanism for metastatic dissemination [41]. These and other studies focused on the molecular underpinnings serve to further define the tumor subtype and raise optimism for the development of more effective means of detection and treatment.

\section{Acknowledgements}

The authors would like to acknowledge the support of Ekaterina Olkhov-Mitsel PhD and Jananie Baskaran with the preparation of this manuscript.

\section{Statement of Ethics}

The study was reviewed and approved by the Research Ethics Boards of the University Health Network (\#15-9591) and the Sunnybrook Health Sciences Centre (\#305-2018). Written consent of the study subjects was waived.

\section{Conflict of Interest Statement}

None of the authors have relevant conflicts of interest.

\section{Funding Sources}

The authors did not receive any funding.

\section{Author Contributions}

All authors made substantial contributions to the work, acquisition, analysis, or interpretation of the data; participated in manuscript revision and approval of the final version to be published; and agreed to be accountable for all aspects of the work.

\section{References}

1 Wilbur DC, Colgan TJ, Ferenczy AS, Hirschowitz L, Loening T, McCluggage WG, et al. Glandular tumours and precursors. In: Kurman RJ, Carcangiu ML, Herrington CS, Young RH, editors. WHO classification of tumours of female reproductive organs. 4th ed. Lyon: International Agency for Research on Cancer; 2014. p. 183-206.

2 Mikami Y. Gastric-type mucinous carcinoma of the cervix and its precursors - histori- cal overview. Histopathology. 2020;76(1): $102-11$.

3 Stolnicu S, Barsan I, Hoang L, Patel P, Terinte C, Pesci A, et al. International Endocervical Adenocarcinoma Criteria and Classification (IECC): a new pathogenetic classification for invasive adenocarcinomas of the endocervix. Am J Surg Pathol. 2018;42(2):214-26.

4 Tsuda H, Mikami Y, Kaku T, Akiyama F, Hasegawa T, Okada S, et al. Interobserver vari- ation in the diagnosis of adenoma malignum (minimal deviation adenocarcinoma) of the uterine cervix. Pathol Int. 2003;53(7):440-9.

5 Tsuda H, Mikami Y, Kaku T, Hasegawa T, Akiyama F, Ohishi Y, et al. Reproducible and clinically meaningful differential diagnosis is possible between lobular endocervical glandular hyperplasia and "adenoma malignum" based on common histopathological criteria. Pathol Int. 2005;55(7):412-8. 
6 Banno K, Kisu I, Yanokura M, Masuda K, Ueki A, Kobayashi Y, et al. Hereditary gynecological tumors associated with Peutz-Jeghers syndrome (review). Oncol Lett. 2013;6(5): $1184-8$.

7 Kuragaki C, Enomoto T, Ueno Y, Sun H, Fujita M, Nakashima R, et al. Mutations in the STK11 gene characterize minimal deviation adenocarcinoma of the uterine cervix. Lab Invest. 2003;83(1):35-45.

8 Garg S, Nagaria TS, Clarke B, Freedman O, Khan Z, Schwock J, et al. Molecular characterization of gastric-type endocervical adenocarcinoma using next-generation sequencing. Mod Pathol. 2019;32(12):1823-33.

9 Kawakami F, Mikami Y, Sudo T, Fujiwara K, Hirose T, Itoh T. Cytologic features of gastrictype adenocarcinoma of the uterine cervix. Diagn Cytopathol. 2015;43(10):791-6.

10 Li G, Jiang W, Gui S, Xu C. Minimal deviation adenocarcinoma of the uterine cervix. Int J Gynaecol Obstet. 2010;110(2):89-92.

11 Yoden E, Mikami Y, Fujiwara K, Kohno I, Imajo Y. Florid endocervical glandular hyperplasia with pyloric gland metaplasia: a radiologic pitfall. J Comput Assist Tomogr. 2001; 25(1):94-7.

12 Takatsu A, Shiozawa T, Miyamoto T, Kurosawa K, Kashima H, Yamada T, et al. Preoperative differential diagnosis of minimal deviation adenocarcinoma and lobular endocervical glandular hyperplasia of the uterine cervix: a multicenter study of clinicopathology and magnetic resonance imaging findings. Int J Gynecol Cancer. 2011;21(7):128796.

13 Fulmer CG, Hoda RS, Pirog EC, Park KJ, Holcomb K. Cytomorphology of gastric-type cervical adenocarcinoma on a ThinPrep Pap test: report of a p16-positive tumor case. Diagn Cytopathol. 2016;44(8):710-3.

14 Hissong E, Yoxtheimer LM, Pacecca A, Hoda RS. Cytology of minimal deviation endocervical adenocarcinoma (adenoma malignum) on a ThinPrep Pap test. Diagn Cytopathol. 2016; 44(6):552-5.

15 Lu S, Shen D, Zhao Y, Kang N, Wang X. Primary endocervical gastric-type adenocarcinoma: a clinicopathologic and immunohistochemical analysis of 23 cases. Diagn Pathol. 2019;14(1):72.

16 Chun Y, Kim H, Hong S. Cytologic features of gastric-type mucinous carcinoma of the uterine cervix. Cytopathology. 2018;29(Suppl 1): 26-135.

17 Turashvili G, Morency EG, Kracun M, DeLair DF, Chiang S, Soslow RA, et al. Morphologic features of gastric-type cervical adenocarcinoma in small surgical and cytology specimens. Int J Gynecol Pathol. 2019;38(3):26375.
18 Kojima A, Mikami Y, Sudo T, Yamaguchi S, Kusanagi $\mathrm{Y}$, Ito $\mathrm{M}$, et al. Gastric morphology and immunophenotype predict poor outcome in mucinous adenocarcinoma of the uterine cervix. Am J Surg Pathol. 2007;31(5): 664-72.

19 Karamurzin YS, Kiyokawa T, Parkash V, Jotwani AR, Patel P, Pike MC, et al. Gastric-type endocervical adenocarcinoma: an aggressive tumor with unusual metastatic patterns and poor prognosis. Am J Surg Pathol. 2015; 39(11):1449-57.

20 Nucci MR, Clement PB, Young RH. Lobular endocervical glandular hyperplasia, not otherwise specified: a clinicopathologic analysis of thirteen cases of a distinctive pseudoneoplastic lesion and comparison with fourteen cases of adenoma malignum. Am J Surg Pathol. 1999;23(8):886-91.

21 Mikami Y, Hata S, Fujiwara K, Imajo Y, Kohno I, Manabe T. Florid endocervical glandular hyperplasia with intestinal and pyloric gland metaplasia: worrisome benign mimic of "adenoma malignum”. Gynecol Oncol. 1999; 74(3):504-11.

22 Mikami Y, Kiyokawa T, Hata S, Fujiwara K, Moriya T, Sasano H, et al. Gastrointestinal immunophenotype in adenocarcinomas of the uterine cervix and related glandular lesions: a possible link between lobular endocervical glandular hyperplasia/pyloric gland metaplasia and "adenoma malignum". Mod Pathol. 2004;17(8):962-72.

23 Talia KL, Stewart CJR, Howitt BE, Nucci MR, McCluggage WG. HPV-negative gastric type adenocarcinoma in situ of the cervix: a spectrum of rare lesions exhibiting gastric and intestinal differentiation. Am J Surg Pathol. 2017;41(8):1023-33.

24 Yuan CT, Lin MC, Kuo KT, Wang TH, Mao TL. Gastric-type adenocarcinoma in situ of uterine cervix: cytological and histopathological features of two cases. Virchows Arch. 2016;469(3):351-6.

25 Okuyama R, Hashimoto H, Miura T, Sugita M, Arai M, Tsunoda H, et al. Two cases of adenocarcinoma in situ arising in lobular endocervical glandular hyperplasia indicating localization of mucin on the cluster surface as an early cytological finding of malignant transformation. Diagn Cytopathol. 2017; 45(9):842-7.

26 Ishii K, Katsuyama T, Ota H, Watanabe T, Matsuyama I, Tsuchiya S, et al. Cytologic and cytochemical features of adenoma malignum of the uterine cervix. Cancer. 1999;87(5):24553.

27 Hata S, Mikami Y, Manabe T. Diagnostic significance of endocervical glandular cells with "golden-yellow" mucin on pap smear. Diagn Cytopathol. 2002;27(2):80-4.

28 Omori M, Kondo T, Nakazawa K, Tagaya H, Ohgi M, Fukasawa H, et al. Interpretation of endocervical cells with gastric-type mucin on Pap smears. Am J Clin Pathol. 2018;150(3): 259-66.
29 Yamanoi K, Ishii K, Tsukamoto M, Asaka S, Nakayama J. Gastric gland mucin-specific Oglycan expression decreases as tumor cells progress from lobular endocervical gland hyperplasia to cervical mucinous carcinoma, gastric type. Virchows Arch. 2018;473(3): 305-11.

30 Granter SR, Lee KR. Cytologic findings in minimal deviation adenocarcinoma (adenoma malignum) of the cervix. A report of seven cases. Am J Clin Pathol. 1996;105(3):327-33.

31 Stelow EB, Pambuccian SE, Bardales RH, Debol SM, Mallery S, Lai R, et al. The cytology of pancreatic foamy gland adenocarcinoma. Am J Clin Pathol. 2004;121(6):893-7.

32 Stewart CJ, Frost F, Leake R, Mohan GR, Tan J. Foamy gland changes in gastric-type endocervical neoplasia. Pathology. 2015;47(7): 653-8.

33 Talia KL, McCluggage WG. The developing spectrum of gastric-type cervical glandular lesions. Pathology. 2018;50(2):122-33.

34 Hashi A, Yuminamochi T, Xu JY, Kondo T, Katoh R, Hoshi K. Intranuclear cytoplasmic inclusion is a significant diagnostic feature for the differentiation of lobular endocervical glandular hyperplasia from minimal deviation adenocarcinoma of the cervix. Diagn Cytopathol. 2008;36(8):535-44.

35 Kaku T, Enjoji M. Extremely well-differentiated adenocarcinoma ("adenoma malignum") of the cervix. Int J Gynecol Pathol. 1983;2(1):28-41.

36 Kudo R, Sagae S, Hayakawa O, Kusanagi T, Hashimoto M. The cytological features and DNA content of cervical adenocarcinoma. Diagn Cytopathol. 1987;3(3):191-7.

37 Szyfelbein WM, Young RH, Scully RE. Adenoma malignum of the cervix. Cytologic findings. Acta Cytol. 1984;28(6):691-8.

38 Vogelsang PJ, Nguyen GK, Honoré LH. Exfoliative cytology of adenoma malignum (minimal deviation adenocarcinoma) of the uterine cervix. Diagn Cytopathol. 1995;13(2):146-50.

39 Wada T, Ohishi Y, Kaku T, Aman M, Imamura $\mathrm{H}$, Yasutake N, et al. Endocervical adenocarcinoma with morphologic features of both usual and gastric types: clinicopathologic and immunohistochemical analyses and high-risk HPV detection by in situ hybridization. Am J Surg Pathol. 2017;41(5):696-705.

40 Pirog EC, Park KJ, Kiyokawa T, Zhang X, Chen W, Jenkins D, et al. Gastric-type adenocarcinoma of the cervix: tumor with wide range of histologic appearances. Adv Anat Pathol. 2019;26(1):1-12.

41 Vasseur D, Lopez J, Croce S, Tondeur G, Bonin L, Descotes F, et al. Transcriptome profiling of gastric-type endocervical adenocarcinomas identifies key signaling pathways for tumor progression. Gynecol Oncol. 2020; 157(3):775-82.

Schwock/Starova/Khan/Mirkovic/ Parra-Herran/Ko/Rouzbahman/Ghorab 